\title{
Survivin suppression through STAT3/ $\beta$-catenin is essential for resveratrol-induced melanoma apoptosis
}

\author{
HABIBIE $^{1}$, SATORU YOKOYAMA ${ }^{1}$, SHERIF ABDELHAMED ${ }^{1}$, SURESH AWALE ${ }^{2}$, \\ HIROAKI SAKURAI $^{1,3}$, YOSHIHIRO HAYAKAWA ${ }^{1}$ and IKUO SAIKI $^{1}$ \\ ${ }^{1}$ Division of Pathogenic Biochemistry, Institute of Natural Medicine, \\ ${ }^{2}$ Frontier Research Core for Life Sciences, ${ }^{3}$ Department of Cancer Cell Biology, \\ Graduate School of Medicine and Pharmaceutical Science, \\ University of Toyama, Toyama 930-0194, Japan
}

Received February 26, 2014; Accepted April 25, 2014

DOI: 10.3892/ijo.2014.2480

\begin{abstract}
Although many chemotherapies have been developed for melanomas, successful therapy would be aided by the identification of intrinsic mechanisms that are crucial for melanoma survival. Here, we used resveratrol, a phytoalexin, as an anti-melanoma reagent. Applying resveratrol to various human and murine melanoma cell lines, we show that survivin is essential for melanoma survival in vitro and in vivo and is targeted by resveratrol. Furthermore, we identify the downregulation of survivin transcription by resveratrol through the suppression of $\beta$-catenin and STAT3. In addition, overexpression of survivin protects melanoma cells from resveratrol-induced apoptosis. Collectively, these studies establish that targeting survivin could provide an opportunity to treat melanoma patients.
\end{abstract}

\section{Introduction}

Although melanoma only comprises $<10 \%$ of all forms of skin cancer; it is responsible for the majority of skin cancer-related deaths (1-4). In 2013, it was estimated that 76,690 new cases of melanoma would be diagnosed in the United States, and that 9,480 people would die of this disease (5). When diagnosed in the early stage and when limited to the skin, melanoma is curable; however, in the case of metastatic melanoma, the prognosis is very poor with a 5 -year survival rate of $<20 \%$. Recently, vemurafenib, a BRAF V600E inhibitor, was found to be an attractive drug for melanoma patients and to produce clinical responses in the majority of melanoma patients (6); however, the responses are variable and all patients eventually relapse. The available medicines for melanoma patients are

Correspondence to: Dr Satoru Yokoyama, Division of Pathogenic Biochemistry, Institute of Natural Medicine, University of Toyama, 2630 Sugitani, Toyama 930-0194, Japan

E-mail: yokoyama@inm.u-toyama.ac.jp

Key words: resveratrol, melanoma, survivin therefore not significantly effective (7), and a new strategy is required.

Resveratrol, a phytoalexin originating from plants such as grapes, is an attractive compound for cancer treatment and has a beneficial effect on various diseases, including cardiovascular disease (8), diabetes (9) and cancer (10). Subsequent preclinical and clinical studies reported that resveratrol has both anti-proliferative and anti-metastatic activity in leukemia, breast cancer, and colon cancer (11). Even though resveratrol is known to induce apoptosis in melanoma cells through the suppression of anti-apoptotic proteins such as BCL-2, BCL-xL, survivin (12-19), the responsible molecules and the detailed mechanism have not been fully elucidated.

In the present investigation we showed that resveratrol induces apoptosis in all human and murine melanoma specimens used, even though they were genetically and phenotypically different. Furthermore, we determined for the first time that the transcriptional suppression of survivin is essential for resveratrol-induced apoptosis. We also identified the inhibition of both tumor growth and survivin expression in vivo by resveratrol. Collectively, these results strongly suggest that targeting survivin by reagents such as resveratrol could provide an opportunity to treat genetically varied melanoma patients.

\section{Materials and methods}

Reagents and plasmid preparation. Resveratrol (Tokyo Chemical Industry, Tokyo, Japan) was dissolved in DMSO to make a 100-mM stock solution. pcDNA3.1-polyP was a kind gift from Dr D.E. Fisher (Massachusetts General Hospital, Boston, MA, USA). Survivin cDNA was subcloned into the BamHI-XhoI site of pcDNA3.1 after RT-PCR from cDNA of normal human melanocytes.

Cell culture. UACC257, SK-MEL-28, SK-MEL-2, UACC62, M14, MeWo, SK-MEL-5 cell lines were kind gifts for Dr D.E. Fisher and B16-BL6 cell lines were from Dr I.J. Fidler (MD Anderson Cancer Center, Houston, TX, USA). UACC257, UACC62, M14, MeWo cells were cultured in RPMI-1640. SK-MEL-28, SK-MEL-2, and SK-MEL-5 were cultured in 
Dulbecco's modified Eagle's medium (DMEM), and B16-BL6 cells were cultured in Eagle's minimal essential medium (EMEM). All of the media were supplemented with $2 \mathrm{mM}$ L-glutamine, $10 \%$ fetal bovine serum, $100 \mathrm{U} / \mathrm{ml}$ penicillin, and $100 \mu \mathrm{g} / \mathrm{ml}$ streptomycin. The cells were maintained at $37^{\circ} \mathrm{C}$ in a humidified atmosphere of $5 \% \mathrm{CO}_{2}$.

For the establishment of stable survivin-expressing cells, SK-MEL-28 cells were transfected with pcDNA3.1-polyP as a vector control, or pcDNA3.1-HA/survivin vector and selected with $500 \mu \mathrm{g} / \mathrm{ml} \mathrm{G} 418$ over a period of 6 weeks.

WST-1 assay. Cell viability was quantified using the cell proliferation reagent WST-1 (Dojindo, Kumamoto, Japan). Melanoma cells were seeded and then incubated for $24 \mathrm{~h}$. Resveratrol-containing medium was added to the well at the indicated concentrations. After the indicated incubation time, WST-1 solution was added and absorbance was measured at $450 \mathrm{~nm}$ using a microplate reader. Cells viability was determined as percent viability compared with the control.

Western blot analysis. Whole cell lysates were prepared as described previously (20). Primary antibodies used were specific to caspase-3, poly (ADP-ribose) polymerase (PARP), BCL-2, BCL-xL, XIAP, Survivin, BCL2A1, MCL-1, STAT3, p-STAT3, $\beta$-catenin, p53 (Cell Signaling Technology, Beverly, MA, USA), specific to hemagglutinin (HA) (Roche, Indianapolis, IN, USA) and specific to $\beta$-actin (Santa Cruz Biotechnology, Santa Cruz, CA, USA). All antibodies were used at $\times 2,000$ dilution.

Annexin $V$ and dead cell assay. Apoptotic cell number was determined using the MUSE Annexin V and Dead Cell kit (Merck KGaA, Darmstadt, Germany) according to the manufacturer's instructions. Briefly, after cells were treated with resveratrol, all cells were collected and diluted with phosphate-bufferd saline (PBS) containing $1 \%$ bovine serum albumin (BSA) as a dilution buffer to a concentration of $5 \times 10^{5}$ cells $/ \mathrm{ml}$. The cell suspension $(100 \mathrm{ml})$ was then added to $100 \mu 1$ MUSE Annexin $\mathrm{V}$ and dead cell reagent (2X dilution), incubated for $20 \mathrm{~min}$ at room temperature, and analyzed using the MUSE Cell Analyzer.

Real-time PCR. Expression of survivin mRNA was quantitatively determined by real-time PCR on an ABI PRISM 7300 sequence detection system (Life Technologies Corp., Carlsbad, CA, USA). Total RNAs were prepared using the RNeasy Plus Mini kit (Qiagen, Hilden, Germany). Expression level of survivin mRNA was normalized to the $\beta$-actin gene. The primers used were: 5'-TGC CTG GCA GCC CTT TC-3' (sense) and 5'-CCT CCA AGA AGG GCC AGT TC-3' (antisense) for survivin $\mathrm{mRNA}$ and $5^{\prime}$-GCA CAG AGC CTC GCC TT-3' (sense) and 5'-GTT GTC GAC GAC GAG CG-3' (antisense) for $\beta$-actin mRNA.

Chromatin immunoprecipitation assay (ChIP). ChIP assays were performed as described previously (21). The antibody used was anti-polymerase II serine 2 phosphorylation (Abcam, Cambridge, MA, USA). The primers were: 5'-GCA GTT CTG GTA ACG GTG ATA G-3' (sense) and 5'-GGG CAG AGA AGG GCA TTA TT-3' (antisense) for the survivin gene region,
5'-GAC ATT GAT GGA GAC GGT AAG G-3' (sense) and 5'-ATA GCC AGG ACT AGA CAG AAG T-3' (antisense) for the THBSl gene region, and 5'-CAT CCT CAC CCT GAA GTA CCC-3' (sense) and 5'-TAG AAG GTG TGG TGC CAG ATT-3' (antisense) for the $\beta$-actin gene region.

Animal model. C57BL/6 mice (6-week-old) were purchased from Japan SLC Inc. (Hamamatsu, Japan). The study was conducted in accordance with the standards established by the Guidelines for the Care and Use of Laboratory Animals and approved by the ethics committee of the University of Toyama (permit no. A2012INM-6). Tumor inoculation was performed under isoflurane anesthesia, and all efforts were made to minimize suffering. The B16-BL6 cells were inoculated s.c $\left(2.5 \times 10^{5}\right.$ cells $/ 100 \mu 150 \%$ Matrigel in PBS/ mice) into the flank of anesthetized mice. Mice in each group received resveratrol in $0.5 \%$ carboxymethyl cellulose solution $(100 \mathrm{mg} / \mathrm{kg} / \mathrm{day})$ or vehicle by oral administration every day. The tumor volume was assessed every two days starting from day 9 . The primary tumor was measured using a caliper square along the longer (a) and shorter (b) axis, and tumor volume was calculated by the following formula: tumor volume $\left(\mathrm{mm}^{3}\right)=\mathrm{ab}^{2} / 2$.

Statistical analysis. The results are expressed as the mean \pm standard deviation. Statistical significance $(\mathrm{p}<0.05)$ was evaluated by either Student's two-tailed t-test or one-way ANOVA followed by the Bonferroni post-hoc test, comparing the results to the control.

\section{Results}

Resveratrol induces apoptosis in melanoma cells. To examine the cytotoxicity of resveratrol against melanoma, seven human melanoma cell lines with various genetic backgrounds (Table I) were treated with resveratrol. As summarized in Table I, all the human melanoma cell lines tested showed a similar $\mathrm{IC}_{50}$ range (120.4-257.0 $\left.\mu \mathrm{M}\right)$ even with different genetic backgrounds; therefore, there seemed to be no specific genetic requirement for the efficacy of resveratrol in melanoma cell lines. Because of their different backgrounds, further experiments were performed using UACC257, SK-MEL-28, and SK-MEL-2 cells. The cytotoxicity of resveratrol against human melanoma cell lines was also observed in a dose-dependent manner and a similar result was confirmed in murine B16-BL6 cells (Fig. 1A). To determine whether the cytotoxicity is mediated through canonical apoptotic pathways, we next assessed the biomarkers for apoptotic cell death. As shown in Fig. 1B and $C$, resveratrol induced the cleavage of caspase- 3 and PARP in both human and murine melanoma cell lines and subsequently induced Annexin-V-positive apoptotic cells in a dose-dependent manner. Collectively, these results indicate that resveratrol showed cytotoxicity in human and murine melanoma cells through the induction of canonical apoptotic pathways regardless of their genetic background.

Resveratrol suppresses survivin expression in melanoma cells. In order to determine whether apoptosis by resveratrol could be mediated via the suppression of anti-apoptotic 
Table I. Oncogenic mutation and $\mathrm{IC}_{50}$ of resveratrol in human melanoma cells.

\begin{tabular}{|c|c|c|c|c|c|}
\hline Melanoma cells & BRAF & NRAS & p53 & PTEN & $\mathrm{IC}_{50}(\mu \mathrm{M})$ \\
\hline UACC257 & V600E & wt & wt & wt & 143.4 \\
\hline SK-MEL-28 & V600E & wt & L145R & wt & 257.0 \\
\hline SK-MEL-2 & wt & Q61R & G245S & wt & 159.5 \\
\hline M14 & V600E & wt & G266E & wt & 232.8 \\
\hline MeWo & wt & wt & Q317X/E258K & wt & 120.4 \\
\hline SK-MEL-5 & V600E & wt & wt & wt & 158.8 \\
\hline UACC62 & V600E & wt & wt & p245fs5 & 218.8 \\
\hline
\end{tabular}
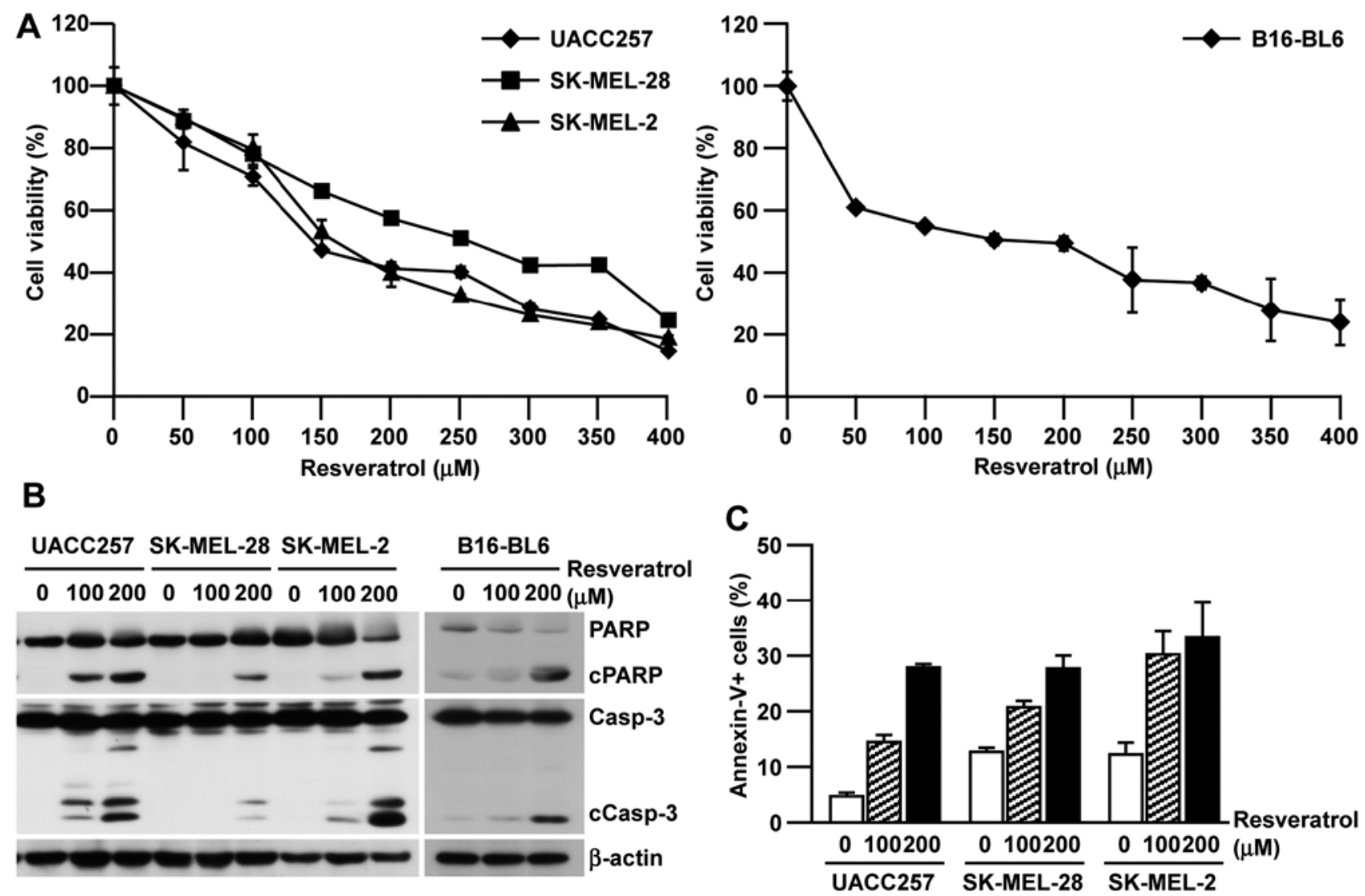

Figure 1. Resveratrol induces apoptosis in melanoma cells. (A) Human melanoma cells (upper panel) or murine melanoma cells (lower panel) were treated with the indicated concentrations of resveratrol for $24 \mathrm{~h}$, and cell viability was determined by the WST-1 assay. Data are shown as the mean \pm SD of three independent experiments. (B) Protein expressions of apoptotic markers were determined with antibodies against caspase-3 and PARP. Other conditions were similar to (A). (C) Total apoptotic cells were determined by Annexin V and dead cell assays. Data are shown as the mean \pm SD of three independent experiments. Other conditions were similar to (A). ${ }^{* *} \mathrm{p}<0.01$ vs. untreated cells by two-way analysis of variance (ANOVA) followed by the Bonferroni post-hoc test.

proteins, the expression levels of BCL-2, BCL-xL, XIAP, survivin, BCL2A1, and MCL-1 were determined after treatment with resveratrol (Fig. 2). While suppression of BCL-2, XIAP, and BCL-xL was observed only in SK-MEL-2 cells, not in SK-MEL-28 and UACC257 cells, the expressions of survivin, BCL2A1 and MCL-1 were strongly suppressed in all three human melanoma cell lines. Since only survivin was consistently downregulated in murine B16-BL6 melanoma cells as well as human melanoma cell lines, we decided to further focus on the role of survivin in the anticancer efficacy of resveratrol in melanoma cells as a conserved mechanism in both humans and mice.

Requirement of survivin suppression for resveratrol-induced apoptosis. To investigate the requirement of survivin for resveratrol-induced apoptosis in melanoma, we established hemagglutinin (HA)-tagged survivin-overexpressing cells (SK-MEL-28/Survivin) and control cells (SK-MEL-28/Vector, Fig. 3A) to test the pro-apoptotic effect of resveratrol. As shown in Fig. 3B, the induction of Annexin-V-positive apoptotic cells 


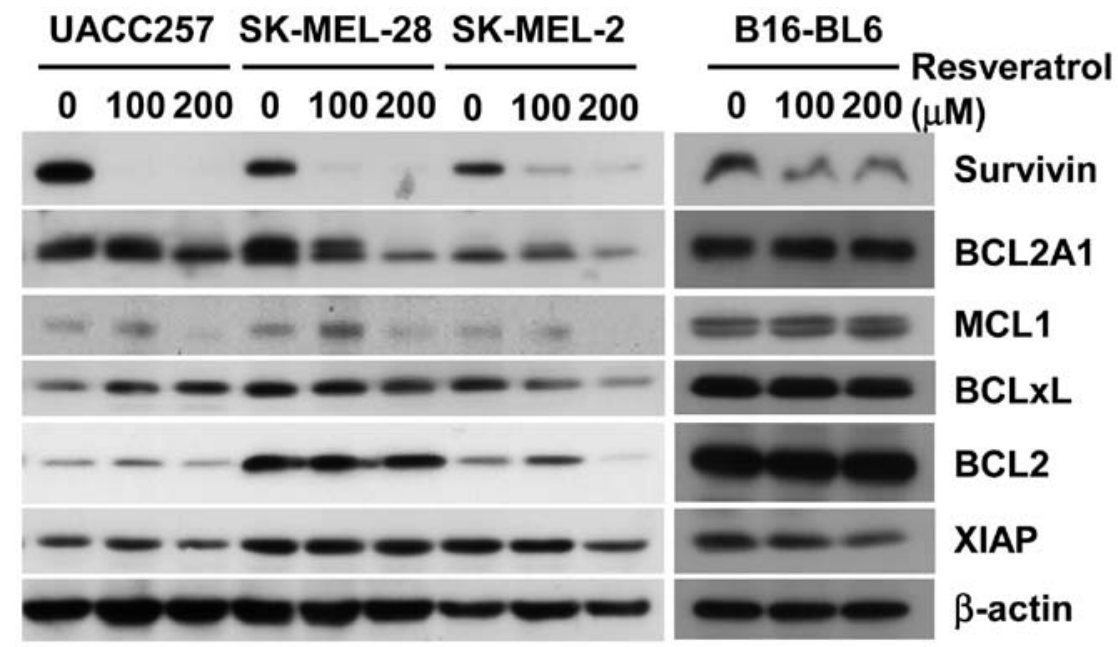

Figure 2. Resveratrol suppresses survivin expression in melanoma cells. Human melanoma cells (left panel) or murine melanoma cells (right panel) were treated with the indicated concentrations of resveratrol for $24 \mathrm{~h}$. Anti-apoptotic proteins were examined with antibodies against survivin, BCL2A1, MCL1, BCLXL, BCL2 and XIAP.
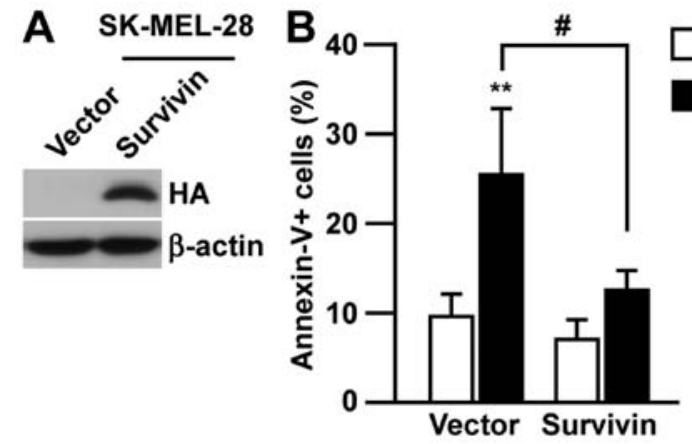

C

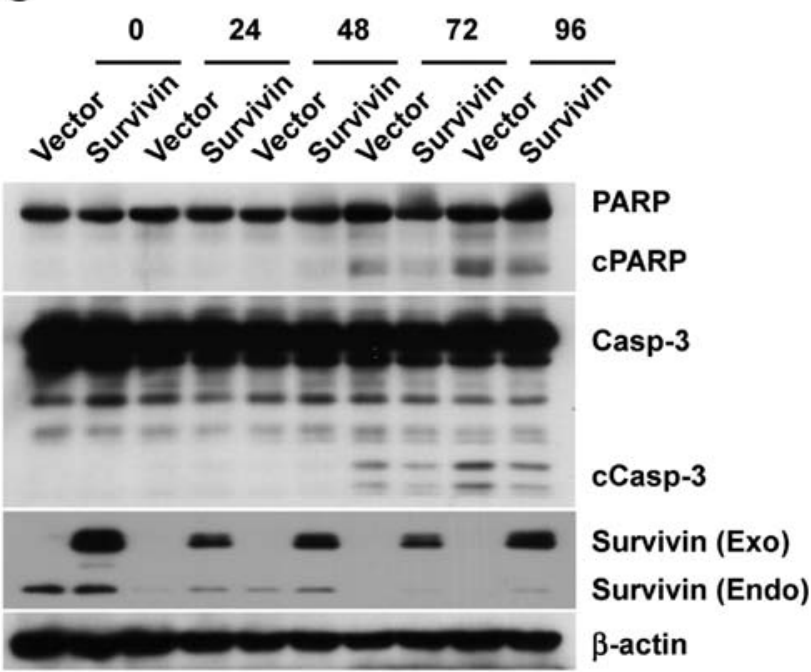

Figure 3. Requirement of survivin suppression for resveratrol-induced apoptosis. (A) Exogenous survivin expression was determined using an antibody against $\mathrm{HA}$ in HA/survivin-overexpressing cells (SK-MEL-28/ Survivin, shown as Survivin) and in vector control cells (SK-MEL-28/ Vector, shown as Vector). (B) SK-MEL-28/Vector or SK-MEL-28/Survivin was treated with $100 \mu \mathrm{M}$ resveratrol for $24 \mathrm{~h}$ and total apoptotic cells were determined by Annexin V and dead cell assays. ${ }^{* *} \mathrm{p}<0.01 \mathrm{vs}$. DMSO-treated cells, and ${ }^{\#} \mathrm{p}<0.05$ vs. SK-MEL-28/Vector with $100 \mu \mathrm{M}$ resveratrol by twoway ANOVA followed by the Bonferroni post-hoc test. (C) SK-MEL-28/ Vector or SK-MEL-28/Survivin was treated with $100 \mu \mathrm{M}$ resveratrol for the indicated time. Protein expressions were determined with antibodies against PARP, caspase- 3 and survivin. was significantly suppressed by overexpressing survivin in SK-MEL-28. Consistent with the reduction in Annexin-Vpositive cells, the overexpression of survivin in SK-MEL-28 reduced the cleavage of PARP and caspase- 3 upon resveratrol treatment (Fig. 3C). This indicated the direct involvement of survivin suppression in resveratrol-induced apoptosis.

Transcriptional regulation of survivin by resveratrol in melanoma cells. In order to determine the molecular mechanism by which resveratrol inhibits survivin expression, we next examined the effect of resveratrol on survivin mRNA expression using real-time RT-PCR and the transcriptional levels of survivin by chromatin immunoprecipitation assay. As shown in Fig. 4A, the expression of survivin mRNA was decreased in all three human melanoma cell lines after resveratrol treatment. Consistently, the occupancy of pol-II S2 in the survivin gene region specifically decreased after resveratrol treatment compared with the $\beta$-actin region or THBSI region, in which THBS1 expression is known to be increased by resveratrol treatment (Fig. 4B) (22). To further investigate which transcription factor is responsible for the suppression of survivin transcription, we next determined the expression of STAT3, $\beta$-catenin, p65 and p53, which are known to be involved in survivin transcription (23). Interestingly, resveratrol strongly inhibited $\beta$-catenin expression and STAT3 phosphorylation in all three melanoma cell lines, but did not show a significant effect on p53 expression (Fig. 4C). Suppression of p65 phosphorylation was only seen in SK-MEL-2 cells, not in SK-MEL-28 or UACC257 cells. Collectively, these results indicate that resveratrol inhibits survivin expression at the transcriptional level through the regulation of $\beta$-catenin and STAT3 pathways.

In vivo antitumor effect of resveratrol in B16-BL6 melanoma model. Finally, we tested the efficacy of resveratrol against highly malignant B16-BL6 melanoma in syngenic immunocompetent C57BL/6 mice. Consistent with our in vitro data, we observed that the administration of resveratrol significantly inhibited B16-BL6 melanoma growth compared to the vehicle 
A

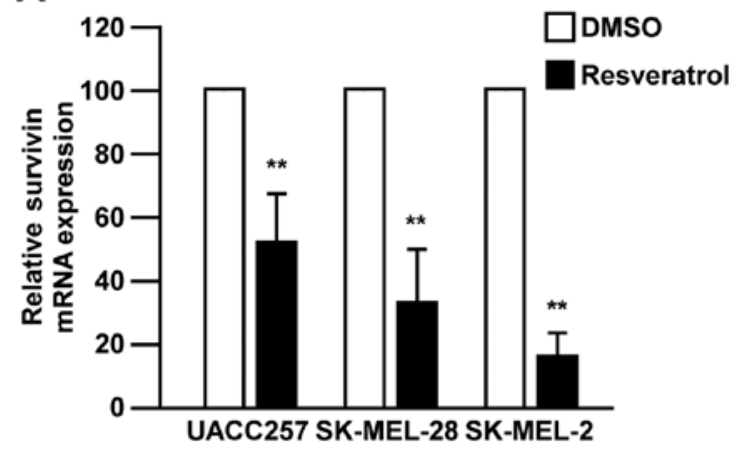

B
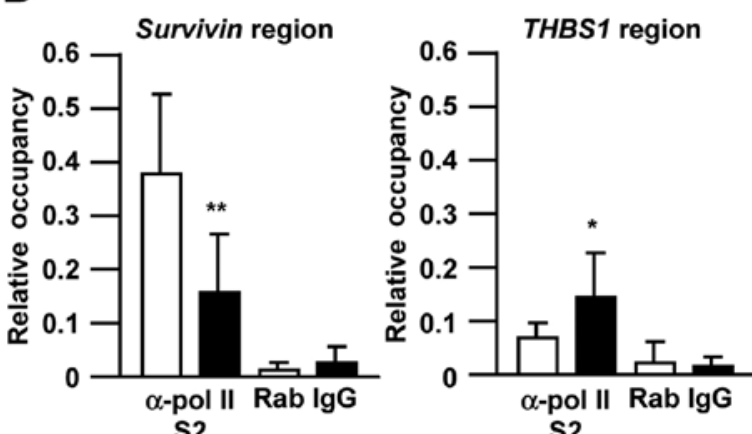

C

UACC257 SK-MEL-28 SK-MEL-2
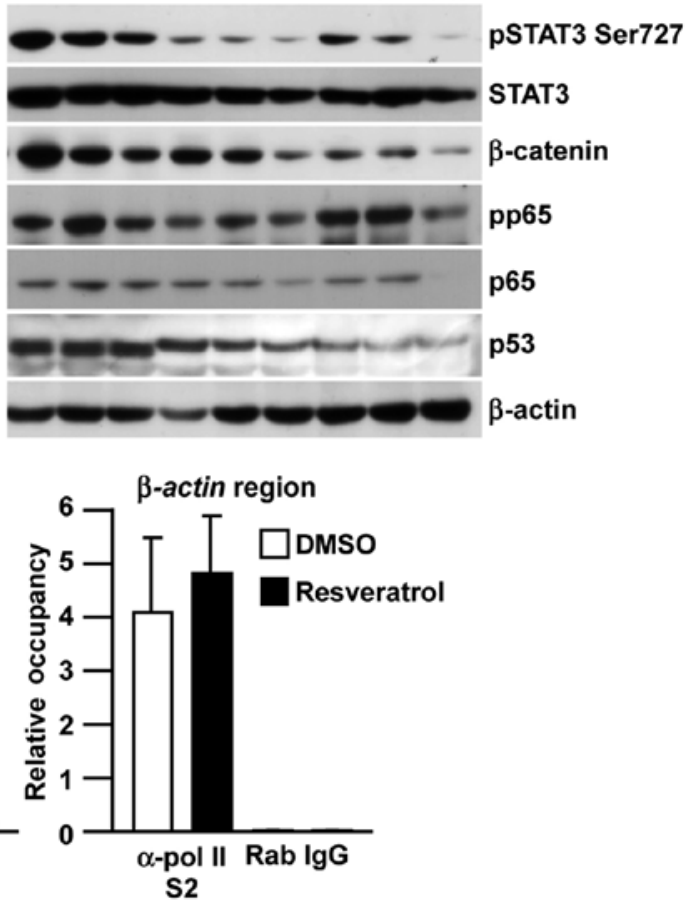

Figure 4. Resveratrol inhibits transcription of survivin in melanoma cells via STAT3 and $\beta$-catenin. (A) Human melanoma cells were treated with $200 \mu \mathrm{M}$ resveratrol for $24 \mathrm{~h}$. Survivin mRNA was quantified by real-time RT-PCR. Data are shown as the mean \pm SD of three independent experiments. ${ }^{* *} \mathrm{p}<0.01$ vs. each cell with DMSO by two-way ANOVA followed by the Bonferroni post-hoc test. (B) SK-MEL-28 cells were treated with $200 \mu \mathrm{M}$ resveratrol for $24 \mathrm{~h}$. Chromatin immunoprecipitation assay was performed by using the antibody against the polymerase II phosphorylated serine 2 site ( $\alpha$-pol II S2). Immunoprecipitated DNA was quantified by real-time PCR using primers specific to the survivin, THBS1 and $\beta$-actin genes. Results are normalized to $1 \%$ input of genomic DNA. Data are shown as the mean \pm SD of three independent experiments. ${ }^{* *}$ p $<0.01$ vs. $\alpha$-pol II S2-precipitated DNA with DMSO by two-way ANOVA followed by the Bonferroni post-hoc test. (C) Protein expressions of transcription factors related to survivin expression were examined with antibodies against STAT3, p-STAT3 ${ }^{\text {ser727, }} \beta$-catenin, p65, p-p65 and p53. Other conditions were the same as in Fig. 2.

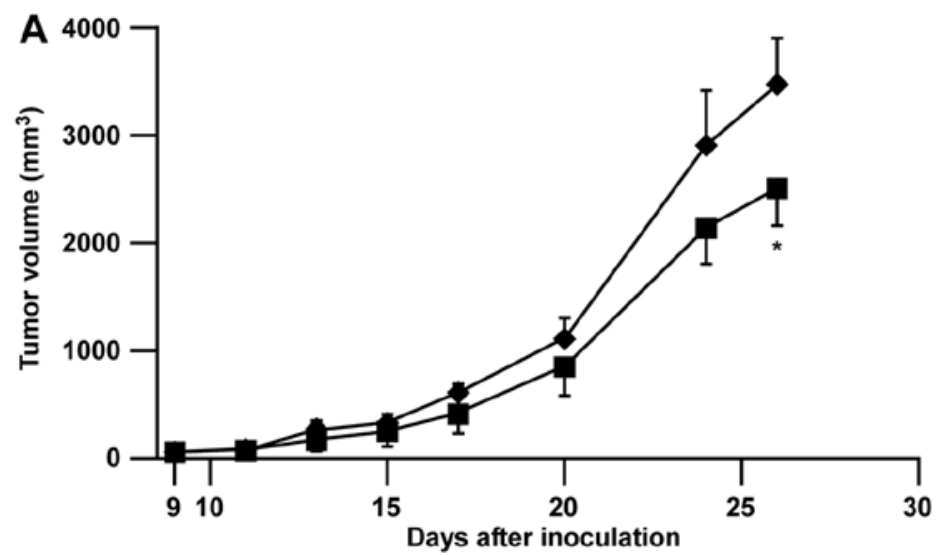

B
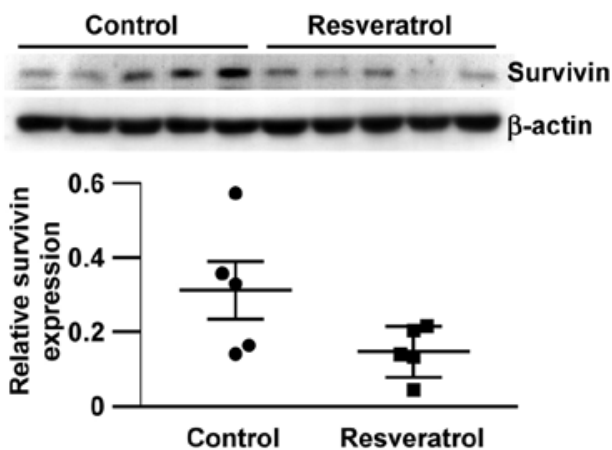

Figure 5. Resveratrol inhibits B16-BL6 melanoma tumor growth in C57BL/6 mice. (A) B16-BL6 cells were s.c. inoculated into mice. Mice received resveratrol in $0.5 \%$ carboxymethyl cellulose solution $(100 \mathrm{mg} / \mathrm{kg} /$ day) or vehicle by oral administration every day. Tumor volume was measured at the indicated times. Data are shown as the mean \pm SEM $(n=5)$. " p $<0.05$ vs. control group at day 26 by two-way ANOVA followed by the Bonferroni post-hoc test. (B) At 14 days after inoculation, tumors were excised and the proteins were extracted from each tumor. Protein expressions were examined with antibodies against survivin. Other conditions were the same as in (A). Lower panel shows the intensities of survivin normalized to $\beta$-actin. Data are represented as the mean $\pm \operatorname{SEM}(n=5)$.

control without any body weight loss (Fig. 5A and data not shown) and further, the reduction of survivin expression in tumors after consecutive treatment with resveratrol (14 days, Fig. 5B).

\section{Discussion}

In this study, we showed that resveratrol induces apoptosis through survivin in various melanoma cell lines in vitro 
without any genetic requirement and further demonstrated the efficacy of resveratrol against melanoma tumor growth in a mouse model. The transcriptional suppression of survivin by resveratrol was mediated through either the $\beta$-catenin or STAT3 pathway.

Although we concluded that survivin was critically involved in resveratrol-induced apoptosis, a substantial level of apoptosis (Fig. 3C) and in vitro growth inhibition (Fig. 5A) were observed in SK-MEL-28/Survivin cells treated with resveratrol. These results may imply some additional mechanisms of resveratrol in melanoma growth inhibition other than the transcriptional suppression of survivin. Indeed, there are several reports regarding the downstream targets of $\beta$-catenin or STAT3 other than survivin that might be related to resveratrol-induced apoptosis. In our results, BCL2A1, MCL-1, and BCL-xL were not consistently regulated by resveratrol in some melanoma cells (Fig. 2). In addition, resveratrol-induced apoptosis was not rescued by overexpression of BCL2A1 in melanoma cells (data not shown), suggesting that BCL2A1, MCL-1 and BCL-xL are unlikely to be responsible for resveratrol-induced apoptosis. The mTOR pathway is also known as an important target of resveratrol in certain cancers $(24,25)$ and is a potential repressor of survivin translation $(26)$. Consistent with previous reports $(24,25)$, we observed the suppression of p70 S6K phosphorylation by resveratrol, one of the downstream molecules in the mTOR pathway in some melanoma cell lines (data not shown); therefore, inhibition of the mTOR pathway might be an alternative mechanism of resveratrol-induced apoptosis in melanoma.

Considering that survivin is known as a therapeutic target for various cancer types such as leukemia, prostate, breast, bladder, colon and melanoma (27-31) and that resveratrol reduced cell viability in all melanoma cell lines regardless of their genetic background in BRAF/NRAS/p53/PTEN (Fig. 1 and Table I), the utility of resveratrol in cancer treatment is feasible. Importantly, survivin expression was higher in melanoma than in nevus and normal skin when we re-analyzed the published microarray data set (32) (data not shown) and survivin expression was also very limited in normal adult tissues (33). In addition, resveratrol could suppress UV damage in normal human keratinocytes, resulting in adverse effects of the prevention of photocarcinogenesis (34). Considering that we did not observe any weight loss in mice with resveratrol in vivo (data not shown), survivin inhibitors including resveratrol could be attractive reagents for cancer therapy regardless of the cancer type. Of note, resveratrol even reduced the cell viability of SK-MEL-5 cell line, which is known to have high $\beta$-catenin activity (35), suggesting that resveratrol might be effective against various target cancer cells by overcoming the high transcriptional activity of $\beta$-catenin or STAT3.

Collectively, these results strongly suggest that targeting survivin with reagents such as resveratrol could provide a new opportunity for melanoma therapy, regardless of the genetic background.

\section{Acknowledgements}

We thank Dr D.E. Fisher (MGH, Boston, MA, USA) for the kind gifts of melanoma cell lines and plasmids, and all members of the Saiki laboratory for discussions and suggestions. This study was supported in part by a Grant-in-Aid for Young Scientists (B) (no. 24700971) (S.Y.), by a Grant-in-aid for Challenging Exploratory Research (24659348) (I.S.) from the Ministry of Education, Culture, Sports, Science, and Technology (Japan), by Kato Memorial Bioscience Foundation (Japan) (S.Y.), and by a Grant-in-Aid for the Cooperative Research Project (I.S.) from the Joint Usage/Research Center (Joint Usage/Research Center for Science-Based Natural Medicine), Institute of Natural Medicine, University of Toyama, in 2013.

\section{References}

1. Chudnovsky Y, Khavari PA and Adams AE: Melanoma genetics and the development of rational therapeutics. J Clin Invest 115: 813-824, 2005.

2. Jemal A, Devesa SS, Fears TR and Hartge P: Cancer surveillance series: changing patterns of cutaneous malignant melanoma mortality rates among whites in the United States. J Natl Cancer Inst 92: 811-818, 2000.

3. Jemal A, Devesa SS, Hartge P and Tucker MA: Recent trends in cutaneous melanoma incidence among whites in the United States. J Natl Cancer Inst 93: 678-683, 2001.

4. Jemal A, Siegel R, Ward E, Hao Y, Xu J and Thun MJ: Cancer statistics, 2009. CA Cancer J Clin 59: 225-249, 2009.

5. Siegel R, Naishadham D and Jemal A: Cancer statistics, 2013. CA Cancer J Clin 63: 11-30, 2013.

6. Flaherty KT, Puzanov I, Kim KB, et al: Inhibition of mutated, activated BRAF in metastatic melanoma. N Engl J Med 363: 809-819, 2010

7. Nashan D, Muller ML, Grabbe S, Wustlich S and Enk A: Systemic therapy of disseminated malignant melanoma: an evidence-based overview of the state-of-the-art in daily routine. J Eur Acad Dermatol Venereol 21: 1305-1318, 2007.

8. Petrovski G, Gurusamy N and Das DK: Resveratrol in cardiovascular health and disease. Ann NY Acad Sci 1215: 22-33, 2011.

9. Szkudelska K and Szkudelski T: Resveratrol, obesity and diabetes. Eur J Pharmacol 635: 1-8, 2010.

10. Signorelli $P$ and Ghidoni R: Resveratrol as an anticancer nutrient: molecular basis, open questions and promises. J Nutr Biochem 16: 449-466, 2005.

11. Aggarwal BB, Bhardwaj A, Aggarwal RS, Seeram NP, Shishodia $S$ and Takada Y: Role of resveratrol in prevention and therapy of cancer: preclinical and clinical studies. Anticancer Res 24: 2783-2840, 2004.

12. Niles RM, McFarland M, Weimer MB, Redkar A, Fu YM and Meadows GG: Resveratrol is a potent inducer of apoptosis in human melanoma cells. Cancer Lett 190: 157-163, 2003.

13. Fulda S and Debatin KM: Sensitization for anticancer druginduced apoptosis by the chemopreventive agent resveratrol. Oncogene 23: 6702-6711, 2004.

14. Ivanov VN, Partridge MA, Johnson GE, Huang SX, Zhou H and Hei TK: Resveratrol sensitizes melanomas to TRAIL through modulation of antiapoptotic gene expression. Exp Cell Res 314: 1163-1176, 2008.

15. Fulda $\mathrm{S}$ and Debatin KM: Sensitization for tumor necrosis factor-related apoptosis-inducing ligand-induced apoptosis by the chemopreventive agent resveratrol. Cancer Res 64: 337-346, 2004.

16. Fang Y, Bradley MJ, Cook KM, Herrick EJ and Nicholl MB: A potential role for resveratrol as a radiation sensitizer for melanoma treatment. J Surg Res 183: 645-653, 2013.

17. Kim MY: Nitric oxide triggers apoptosis in A375 human melanoma cells treated with capsaicin and resveratrol. Mol Med Rep 5: 585-591, 2012.

18. Osmond GW, Augustine CK, Zipfel PA, Padussis J and Tyler DS: Enhancing melanoma treatment with resveratrol. J Surg Res 172: 109-115, 2012.

19. Tak JK, Lee JH and Park JW: Resveratrol and piperine enhance radiosensitivity of tumor cells. BMB Rep 45: 242-246, 2012.

20. Sakurai H, Suzuki S, Kawasaki N, et al: Tumor necrosis factor-alpha-induced IKK phosphorylation of NF-kappaB p65 on serine 536 is mediated through the TRAF2, TRAF5, and TAK1 signaling pathway. J Biol Chem 278: 36916-36923, 2003. 
21. Haq R, Yokoyama S, Hawryluk EB, et al: BCL2A1 is a lineagespecific antiapoptotic melanoma oncogene that confers resistance to BRAF inhibition. Proc Natl Acad Sci USA 110: 4321-4326, 2013.

22. Trapp V, Parmakhtiar B, Papazian V, Willmott L and Fruehauf JP: Anti-angiogenic effects of resveratrol mediated by decreased VEGF and increased TSP1 expression in melanoma-endothelial cell co-culture. Angiogenesis 13: 305-315, 2010.

23. Altieri DC: Survivin, cancer networks and pathway-directed drug discovery. Nat Rev Cancer 8: 61-70, 2008.

24. Zhang J, Chiu J, Zhang H, et al: Autophagic cell death induced by resveratrol depends on the $\mathrm{Ca}(2+) / \mathrm{AMPK} / \mathrm{mTOR}$ pathway in A549 cells. Biochem Pharmacol 86: 317-328, 2013.

25. Jiang $\mathrm{H}$, Shang $\mathrm{X}$, Wu H, et al: Resveratrol downregulates PI3K/ Akt/mTOR signaling pathways in human U251 glioma cells J Exp Ther Oncol 8: 25-33, 2009.

26. Vaira V, Lee CW, Goel HL, Bosari S, Languino LR and Altieri DC: Regulation of survivin expression by IGF-1/mTOR signaling. Oncogene 26: 2678-2684, 2007.

27. Zhao W, Bao P, Qi H and You H: Resveratrol down-regulates survivin and induces apoptosis in human multidrug-resistant SPC-A-1/CDDP cells. Oncol Rep 23: 279-286, 2010.

28. Hayashibara T, Yamada Y, Nakayama S, et al: Resveratrol induces downregulation in survivin expression and apoptosis in HTLV-1-infected cell lines: a prospective agent for adult T cell leukemia chemotherapy. Nutr Cancer 44: 193-201, 2002.
29. Shankar S, Siddiqui I and Srivastava RK: Molecular mechanisms of resveratrol (3,4,5-trihydroxy-trans-stilbene) and its interaction with TNF-related apoptosis inducing ligand (TRAIL) in androgen-insensitive prostate cancer cells. Mol Cell Biochem 304: 273-285, 2007.

30. Singh SK, Moretta D, Almaguel F, Wall NR, De Leon M and De Leon D: Differential effect of proIGF-II and IGF-II on resveratrol induced cell death by regulating survivin cellular localization and mitochondrial depolarization in breast cancer cells. Growth Factors 25: 363-372, 2007.

31. Singh RP, Tyagi A, Sharma G, Mohan S and Agarwal R: Oral silibinin inhibits in vivo human bladder tumor xenograft growth involving down-regulation of survivin. Clin Cancer Res 14: 300-308, 2008

32. Talantov D, Mazumder A, Yu JX, et al: Novel genes associated with malignant melanoma but not benign melanocytic lesions. Clin Cancer Res 11: 7234-7242, 2005.

33. Velculescu VE, Madden SL, Zhang L, et al: Analysis of human transcriptomes. Nat Genet 23: 387-388, 1999.

34. Adhami VM, Afaq F and Ahmad N: Suppression of ultraviolet B exposure-mediated activation of NF-kappaB in normal human keratinocytes by resveratrol. Neoplasia 5: 74-82, 2003.

35. Widlund HR, Horstmann MA, Price ER, et al: Beta-catenininduced melanoma growth requires the downstream target Microphthalmia-associated transcription factor. J Cell Biol 158: 1079-1087, 2002. 